\title{
Geometrical Alignment of Multiple Fabrication Steps for Rapid Prototyping of Microfluidic Paper-Based Analytical Devices
}

\author{
Mohammad Rahbar, Pavel N. Nesterenko, Brett Paull, ${ }^{\circledR}$ and Mirek Macka*(i) \\ School of Physical Sciences and Australian Centre for Research on Separation Science (ACROSS), University of Tasmania, Hobart \\ 7001, Australia
}

\section{Supporting Information}

\begin{abstract}
Three main fabrication steps for microfluidic paper-based analytical devices ( $\mu$ PADs) were fully integrated with accurate geometrical alignment between the individual steps in a simple and rapid manner. A wax printer for creating hydrophobic barriers was integrated with an inexpensive (ca. \$300) electronic craft plotter/cutter for paper cutting, followed by colorimetric reagent deposition using technical pens. The principal shortcoming in the lack of accurate and precise alignment of the features created by these three individual fabrication steps was addressed in this work by developing appropriate alignment procedures during the multistep fabrication process. The wax printing step was geometrically aligned with the following cutting and plotting (deposition) steps in a highly accurate and precise manner using optical scanning function of the plotter/cutter based on registration marks printed on the paper using the wax printer and scanned by the plotter/ cutter. The accuracy and precision of alignment in a two-dimensional plane were quantified by cutting and plotting cross-shaped features and measuring their center coordinates relative to wax printed reference lines. The average accuracy along the $X$ - and $Y$ axis was 0.12 and $0.16 \mathrm{~mm}$ for cutting and 0.19 and $0.17 \mathrm{~mm}$ for plotting, respectively. The potential of this approach was demonstrated by fabricating $\mu \mathrm{PADs}$ for instrument-free determination of cobalt in waters using distance-based readout, with excellent precision $(\% \mathrm{RSD}=5.7$ ) and detection limit $(\mathrm{LOD})$ of $2.5 \mathrm{ng}$ and $0.5 \mathrm{mg} / \mathrm{L}$ (mass and concentration LODs, respectively).
\end{abstract}

\begin{abstract}
$\mathrm{n}$ the past decade, microfluidic paper-based analytical devices 1 ( $\mu$ PADs) have gained attention due to their unique advantages of low-cost, ultimate portability, ease of use, and disposal. $\mu$ PADs are easy-to-use miniaturized analytical devices which are simply fabricated by demarcating defined hydrophilic channels on paper by creating hydrophobic barriers using wax printing or other techniques. The channels then guide liquids such as sample moving by capillary forces, to react with particular chemical reagents deposited into detection zones. ${ }^{1,2}$

In spite of all the advances, fabrication of $\mu \mathrm{PADs}$ still have some limitations. For instance, fabrication methods are still either tedious or can pose challenges in relation to their facile and reproducible fabrication. These impediments hinder the advance of $\mu \mathrm{PADs}$ in broad areas of analytical chemistry. So far, many varieties of $\mu$ PADs with different designs and applications have been reported fabricated by different methods. ${ }^{3}$ These fabrication methods have several (usually three) main steps including creating confined hydrophilic patterns on paper, cutting the required shapes and areas, and deposition of colorimetric reagents on the paper. ${ }^{4,5}$ Each of these steps is usually realized with different techniques. So for creating hydrophilic patterns, several methods have been reported including wax printing, paper cutting and shaping, ink stamping, laser treatment, photolithography, and plotting., ${ }^{4,5}$ Among those various methods, wax printing has been most
\end{abstract}

widely used due to its inherent advantages of low-cost, simplicity, easy and rapid fabrication (only one heating step is needed after printing), and suitability for mass production. ${ }^{6}$

The cutting step has also been accomplished by different devices such as hand-held cutters, hand-held punchers, laser cutters, and electronic craft cutters. ${ }^{4}$ We reported cutting using an electronic craft cutter equipped with standard blades, which offered much more reproducible fabrication compared to hand cutting, and less costly compared to laser cutting. ${ }^{7}$ When it comes to reagent deposition, a few methods have been used for this purpose, including pipetting, brushing, inkjet printing, dipping, and spraying. ${ }^{8}$ We reported deposition of colorimetric reagents on paper using technical pens in an electronic craft plotter/cutter showing great promise due to automated and reproducible deposition. ${ }^{7}$ The use of the craft plotter/cutters in fabrication process of microfluidic devices has increased recently, which is due to the low-cost, flexibility, automated deposition, and simplicity of these instruments. It should be noted that, in most of the reported works, ${ }^{9-16}$ only the cutting feature of the plotter/cutters has been implemented rather than

Received: September 16, 2017

Accepted: November 1, 2017

Published: November 1, 2017 
the plotting. In other words, the craft plotter/cutter has been used to perform very simple cuts upon the paper, vinyl, or other materials without particular demands on precision. The plotting feature has been used only a few times so far for creating hydrophobic barriers on paper by deposition of inks filled in special pens. ${ }^{4,7,17}$

As the three major fabrication tools, namely, wax printing, plotting, and cutting with a plotter/cutter, offer different advantages, they could be used together for fabrication of $\mu \mathrm{PADs}$ based upon their combined use. However, there is no comprehensive method comprising all these three steps accomplished in a simple, low-cost, flexible, reproducible, and rapid way, and importantly, accurately geometrically aligned. In other words, at least one of these three main steps is usually done either by hand or other unreliable ways, which impact the reproducibility and robustness of the whole fabrication process and affects the final results. The paper media has to be moved from one instrument to the next one during the fabrication process which makes it difficult to keep the features fabricated in different steps precisely geometrically aligned.

In this work we show for the first time that a combination of wax printing for making fluidic barriers, digital craft cutter for cutting, and also plotting with the technical pens for reagent deposition, can be accurately aligned to provide a low-cost, simple, rapid, reproducible, and comprehensive fabrication method for prototyping of $\mu$ PADs. Further, we show that the geometrical alignment of this combination of different fabrication steps can be assured by utilizing an optical scanner function of the plotter/cutter. The accuracy and precision of the alignment (deviation in coordinates of fabricated features from theoretical coordinates) were quantified using purposely designed fabrication experiments. As for an analytical demonstration of the developed fabrication method, distancebased $\mu \mathrm{PADs}$ were designed, fabricated and implemented for instrument-free determination of cobalt in water samples, which in itself presents a novel analytical use of $\mu$ PADs as quantitative determination of cobalt using $\mu \mathrm{PADs}$ has not been reported.

\section{EXPERIMENTAL SECTION}

Chemicals and Materials. All chemicals were of analytical reagent grade. Citric acid monohydrate, 1-nitroso-2-naphthol, sodium phosphate dibasic dihydrate, rhodamine 6G, and ethylenediaminetetraacetic acid (EDTA) disodium salt dihydrate were purchased from Sigma-Aldrich. Standard Co(II) solutions were produced by diluting a stock solution of 1000 $\mathrm{mg} / \mathrm{L}$ of the nitrate salt of the element supplied by SigmaAldrich. Water was treated with a Millipore (Bedford, MA, U.S.A.) Milli-Q water purification system. Whatman grade 1 qualitative filter paper with a pore size of $11 \mu \mathrm{m}$ and thickness of $180 \mu \mathrm{m}$ (GE Healthcare Australia Pty. Ltd., NSW, Australia) was used to fabricate the $\mu \mathrm{PADs}$. Transparent laminating film (thickness of $125 \mu \mathrm{m}, \mathrm{GBC}, \mathrm{NSW}$, Australia) was used to laminate the $\mu$ PADs.

Fabrication of the Distance-Based $\mu$ PADs. As shown in Figure S-1, the distance-based microfluidic patterns composed of a straight channel $(1.9 \times 29 \mathrm{~mm})$ and a circular sample zone (3.7 $\mathrm{mm}$ outer diameter) along with scale bars (drawn at 1.0 $\mathrm{mm}$ intervals next to the channel for naked eye measurement of the color change length) were predesigned using the Silhouette Studio software (Figure S-2) and printed on paper by the wax printer. The patterns were printed on both sides of the paper to let the wax penetrate throughout the paper thickness from two sides (top and bottom) after the heating step to prevent any leakage of the sample from the $\mu$ PADs. ${ }^{6,21}$ After that, the sheet was loaded on the adhesive surface of a reusable cutting mat and was placed on the plotter/cutter for cutting out the sample zone as a circular hole in the paper of diameter $3 \mathrm{~mm}$. In the next step, the technical pens were filled with the colorimetric reagents and then inserted in the plotter/cutter to deposit the reagents on the straight channels. Afterward, the sheet was laminated from both sides to give a higher mechanical stability to the final $\mu \mathrm{PAD}$ and also to prevent contamination of the detection areas. Circles (diameter $=4 \mathrm{~mm}$ ) were cut out in the top lamination film (before lamination) by the same cutting as where the filter paper was cut out for introducing sample. The lamination was performed at a temperature of $165{ }^{\circ} \mathrm{C}$ to melt the wax entirely through the thickness of the paper to create consistent hydrophobic barriers. Finally, the fabricated $\mu$ PADs were cut into separate segments with desired sizes using the same cutting system and stored for further use in a sealed plastic bag. Figure S-3 represents the paper media undergoing different fabrication steps.

\section{RESULTS AND DISCUSSION}

Alignment of Multiple Fabrication Steps. The here presented alignment of multiple fabrication method for prototyping of $\mu \mathrm{PADs}$ was composed of three main steps including wax printing of the hydrophobic barriers, cutting out the sample zone and deposition of the colorimetric reagents (Figure 1). As the wax printing was performed by the wax
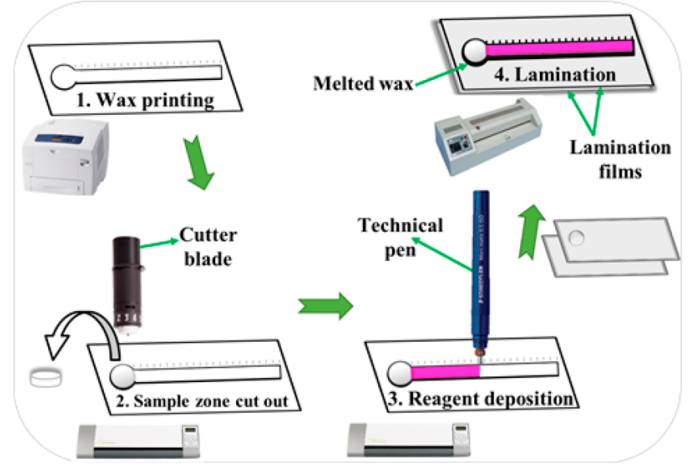

Figure 1. Schematic illustration of the overall fabrication process with geometrically aligned steps for distance-based $\mu \mathrm{PADs}$.

printer while the other two steps were accomplished by the plotter/cutter, it was crucial to keep all the three steps aligned. These two separate devices have different working principles which make it difficult to couple them during the multiple fabrication steps, while keeping all these steps geometrically aligned. Specifically for fabrication of the distance-based $\mu$ PADs, it was essential to perform the cutting and deposition precisely, since we were dealing with very small dimensions during the cutting (circles, inner diameter $=3.3 \mathrm{~mm}$ ) and the deposition (narrow channels, inner width $=1.4 \mathrm{~mm}$ ) steps (Figure S-4). Thus, any misalignment could lead to the cutting or reagent deposition outside of the desired areas. In addition, each of these functions had to be replicated in high numbers (e.g., 170 times for $170 \mu \mathrm{PADs}$ printed on an $\mathrm{A} 4$ size paper) for minimal consumption of paper, as desirable in the case of mass production.

Optical Scanner and "Registration Marks". The working principle of the plotter/cutter is based on electromechani- 


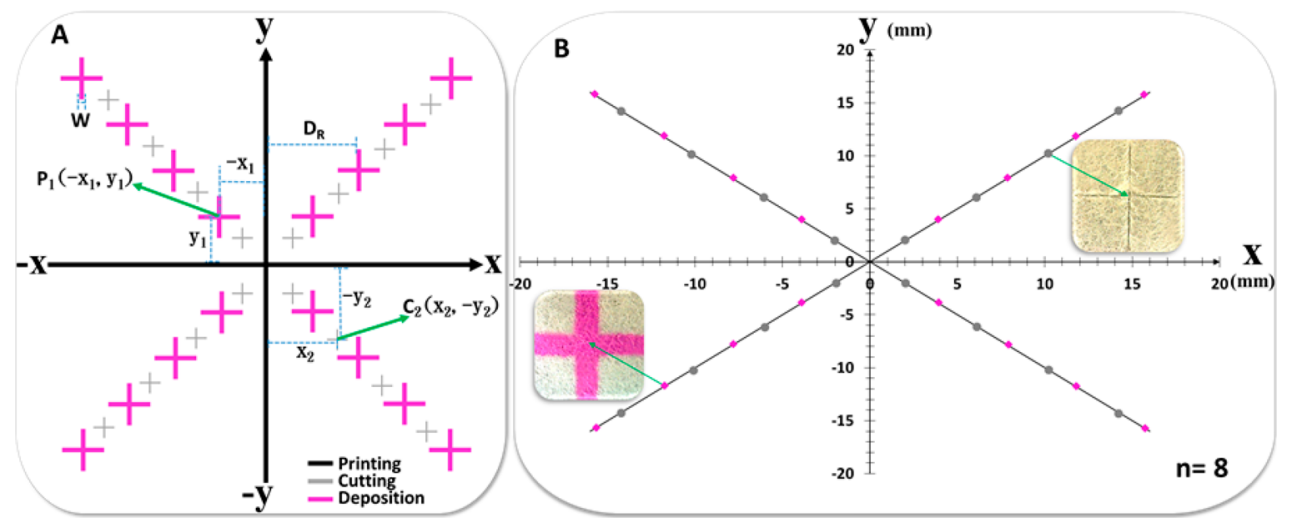

Figure 2. Accuracy and precision of geometrical alignment in multiple fabrication steps. (A) Graphical pattern simulating different fabrication steps of $\mu \mathrm{PADs}$, where the black lines ((black cross) reference lines, $X / Y$-axes) represent the wax printing step, (gray cross) represent the cutting step, and (pink cross) represent the deposition step. $D_{\mathrm{R}}$ is the distance measured by microscope between the edge of plotted lines and the edge of corresponding reference lines, and $W$ is the width of plotted lines. (B) Experimentally determined coordinates of the cut and plotted crosses obtained from the predesigned pattern undergoing $(n=8)$ the corresponding fabrication steps, where the black lines $(\times$ shape $)$ are the expected values for cutting and deposition performances, (gray circles) cutting, and (pink diamonds) deposition coordinates (e.g., $\mathrm{C}_{2}$ and $\mathrm{P}_{1}$ in Figure $2 \mathrm{~A}$ ) obtained after performing either of these steps. Insets are the actual photographs of cut and plotted crosses. For detailed description of the coordinate determination procedures, see Supporting Information.

cally driven movements along the $X / Y$-axes defined by the device (Figure S-5). It is obvious that the $X / Y$-based operation of the machine cannot be reliable enough for such challenging tasks demanding accurate alignment while transferring the paper media from one instrument (wax printer) to another one (plotter/cutter). Fortunately, the latest models of plotter/ cutters such as the Silhouette CAMEO possess an optical scanning feature which is available for highly accurate and precise cutting or plotting, but does not however appear to have been used to-date for fabricating $\mu \mathrm{PADs}$ or in fact any research purpose. Under normal conditions, the optical scanner is deactivated so the machine will function merely based on the $X / Y$-axes, as explained earlier. However, there is an option in the Silhouette Studio software called "Registration Marks", which can be selected while designing the microfluidic patterns, and this is how the scanner function can be activated. These marks are some default patterns which would be printed on the paper media around the workspace border and later will be read by the optical scanner (Figure S-6). Then according to the location of the marks, the software can triangulate the exact position of the lines which are already defined to be cut or plotted. In other words, we can have a very precise control over the performance of the plotter/cutter and so can instruct the instrument to do a task exactly at the locations where desired. Activating the optical scanner by using the "Registration Marks" in the design was the most important action regarding the alignment issue although there were still some other necessary actions and considerations (explained in further details in Supporting Information), which should be taken into account. By applying the optical scanning function along with all those considerations, we could manage to perform the cutting and deposition with a high accuracy and precision in good alignment with the wax printed features. The overall cutting and deposition steps in fabrication of distance-based $\mu$ PADs, are demonstrated in the Supporting Information, Movies S1 and $S 2$, respectively.

Accuracy and Precision of the Alignment. To demonstrate the reliability of the new method for fabrication of $\mu \mathrm{PADs}$, the alignment accuracy of the wax printer with plotter/cutter was investigated. A graphical pattern similar to a simple two-dimensional coordinate system was designed with the Silhouette Studio software to simulate a fabrication process of $\mu \mathrm{PADs}$. As shown in Figure $2 \mathrm{~A}$, this pattern is composed of two reference lines (black lines, $X / Y$-axes, $40 \times 40 \mathrm{~mm}$ ) perpendicular to each other, which were designed to be printed by the wax printer. There are also crosses in this pattern on all four possible areas of a plane which were supposed to be cut (gray color, $3 \times 3 \mathrm{~mm}$ ) and plotted (pink color, $5 \times 5 \mathrm{~mm}$ ) by the plotter/cutter. After performing all these three steps, we measured the coordinates (i.e., centers) of the cut and plotted crosses. The distance of the experimental coordinates from the reference lines compared to the predesigned (theoretical) values provided a numerical representation of the accuracy and precision of the alignment and compatibility of the wax printer with the plotter/cutter. The cutting coordinates (e.g., $C_{2}\left(x_{2}\right.$, $\left.-y_{2}\right)$ in Figure 2A) were measured using a distance-calibrated microscope since the cut lines made by the blade were narrow enough resulting in obvious centers for each cross. The $x$ and $y$ values for each coordinate were obtained by measuring the distance between the centers and the edge of corresponding reference lines (printed $X$ - or $Y$-axis). However, for the plotted crosses we had to determine the center point of cross sections of relatively wide lines. To find out the plotting coordinates (e.g., $P_{1}\left(-x_{1}, y_{1}\right)$ in Figure $2 \mathrm{~A}$ ), we used a simple equation (eq $\mathrm{S} 1)$ to compensate for the distance which the ink penetrates into the paper. Further details in this regard can be found in Supporting Information.

The graph depicted in Figure 2B was obtained from the predesigned pattern undergoing those three steps one after another $(n=8)$. The black lines ( $\times$ shape $)$ in this plot are the expected values for cutting and deposition performances. Gray dots are the cutting and the pink dots are the deposition coordinates obtained after performing either of these steps. All the measured coordinates are well located on the expected lines indicating a highly accurate whole process. Numerical values for the slight deviations of the measured coordinates from the theoretical ones represent the accuracy. The average of these values for 16 coordinates $(\mathrm{n}=8)$ was reported as overall accuracy, which was calculated to be $0.12 \mathrm{~mm}$ and $0.16 \mathrm{~mm}$ for cutting, and $0.19 \mathrm{~mm}$ and $0.17 \mathrm{~mm}$ for plotting, along the $X$ - 
and $Y$-axis, respectively. The fabrication precision as an average standard deviation of individual coordinates was also reported separately along the $X$ - and $Y$-axes, representing the overall precision of the cutting and deposition process (Table 1). Since

Table 1. Accuracy and Precision Values for Alignment of the Cut and Plotted Crosses Performed by the Plotter/Cutter Relative to the Wax Printed Reference Lines

$\begin{array}{ccc}\text { alignment factors }(\mathrm{mm}) & \text { cutting } & \text { deposition } \\ \text { accuracy } X \text {-axis } & 0.12 & 0.19 \\ \text { accuracy } Y \text {-axis } & 0.16 & 0.17 \\ S^{a} X \text {-axis } & 0.04 & 0.05 \\ \text { S Y-axis } & 0.08 & 0.12\end{array}$

${ }^{a} \mathrm{~S}$ is average of standard deviations.

the number of repetitions for obtaining each coordinate was the same $(n=8)$, this average can be regarded as a reliable estimation of the overall standard deviation. As anticipated, the standard deviation along the $Y$-axis was almost two times more than that along the $X$-axis for both cutting and deposition steps. This is to be expected due to the fact that the $Y$-axis is defined by the movement of the whole media via the twisting pinch rollers which can cause some minor drifting during operation. However, the $X$-axis is the result of movement of the pen holder (media is static) over a rail that is likely to be more consistent. These results show that the proposed idea of integrating the wax printer with the plotter/cutter as described in this paper is reliable, highly accurate, and can be implemented for a variety of applications, including prototyping of miniaturized analytical devices such as $\mu \mathrm{PADs}$.

Distance-Based Determination of Cobalt in Waters. In the distance-based measurement, the sample moves forward through straight microfluidic channels and reacts with the deposited colorimetric reagent resulting in a color change thatis observed as a boundary between the reagent alone and the reagent reacted with the analyte. ${ }^{18}$ The length of the color change to that boundary can be related to the concentration of the analyte present in the sample. It has been demonstrated that the distance-based $\mu$ PADs have the potential to be applied for portable, instrument-free and rapid analysis of a variety of analytes. ${ }^{19-24}$ Cobalt is one of the inorganic contaminants the determination of which in natural waters is very important since its excess can cause some serious health problems for humans, animals, plants, and microorganisms. ${ }^{25,26}$ According to the Australian and New Zealand guidelines for fresh and marine water quality, the concentration of cobalt in irrigation waters should not be more than $0.1 \mathrm{mg} / \mathrm{L}$ (short-term use) or 0.05 $\mathrm{mg} / \mathrm{L}$ (long-term use). ${ }^{27}$ Conventional analytical methods for determination of cobalt are costly and time-consuming, and they also need complex equipment and trained operators which are not suitable for on-site analysis. ${ }^{28}$ However, the distancebased $\mu \mathrm{PADs}$ can be applied for rapid, low-cost, instrumentfree, and portable analysis of the water samples.

The developed distance-based $\mu \mathrm{PADs}$ fabricated by this facile method were successfully applied for instrument-free quantification of cobalt in water samples using 1-nitroso-2-naphthol as the metallochromic ligand. The detection chemistry is detailed in the Supporting Information. Determination of standard solutions of Co was investigated in the range of 2.5-100 ng $(0.5-20 \mathrm{mg} / \mathrm{L})$ and the calibration curves were obtained using different distances of color change formed on the $\mu \mathrm{PADs}$ for various concentrations of analytes. The detection limit (LOD), which in distance-based $\mu \mathrm{PADs}$ is considered as the lowest distinguishable length ${ }^{20}$ (usually $0.5 \mathrm{~mm}$ ) of color change along the channel was $2.5 \mathrm{ng}$ and $0.5 \mathrm{mg} / \mathrm{L}$ of $\mathrm{Co}$ as mass and concentration LODs, respectively.

In this work, the unnecessary paper area of the sample zone was cut out (Figure S-7) to improve analytical parameters such as sensitivity and LOD, based on rationale as follows. Even though paper is conventionally considered as a neutral material, but the overall surface charge of the cellulose is negative due to the presence of the carboxyl groups formed by the addition of oxidative reagents to the fibers during papermaking process. The surface of paper will acquire a relatively low cationexchange capacity and can retain metal cations through the electrostatic interactions. ${ }^{29,30}$ Therefore, a portion of the analyte can be adsorbed on the surface of the paper normally available at the sample zone of other $\mu \mathrm{PADs}$ without even reaching to the detection area. This issue will be more considerable when very low concentrations of the metals are being monitored. Removing the sample zone has also been reported in very few previous works ${ }^{20,21}$ with $\mu$ PADs, while the cutting was done by hand-held punching which is not suited for high throughput fabrication and of limited reproducibility. Here we performed this job in a very simple and rapid way with very high precision and speed. This concept was further investigated by comparing two different types of $\mu \mathrm{PADs}$ (with and without cutting the sample zone) for determination of cobalt (Figure S8). As expected, the $\mu \mathrm{PADs}$ without paper in the sample zone exhibited a lower LOD as well as a lower RSD value, and higher sensitivity for detection of cobalt. These results show the importance of sample zone cutting.

Figure 3 represents the performance of the $\mu \mathrm{PADs}$ and associated response curves for detection of Co, after introducing $5 \mu \mathrm{L}$ of standard samples into the inlets of the devices. As illustrated in the corresponding figures, these results represent excellent reproducibility as noted by the standard deviations and error bars. The effect of possible interferences

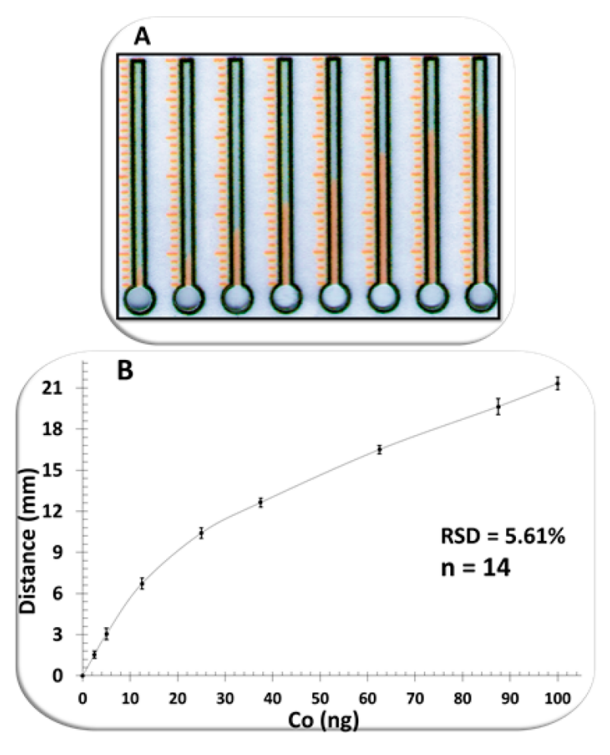

Figure 3. (A) Photograph of distance-based $\mu$ PADs after pipetting 5 $\mu \mathrm{L}$ of Co standard solutions. Contrast and brightness are modified for the sake of better observation. (B) Response curve of different concentrations (2.5-100 ng) of Co to distance-based $\mu$ PADs. Markers reflect the average of 14 repetitive measurements. The error bars represent the standard deviations from the average values. 
on determination of cobalt was also investigated by applying water samples containing Co (5 ppm) and different metal ions ( $\mathrm{Al}, \mathrm{Ca}, \mathrm{Cu}, \mathrm{Fe}, \mathrm{Mg}, \mathrm{Mn}, \mathrm{Ni}, \mathrm{K}, \mathrm{Na}, \mathrm{Pb}$, and $\mathrm{Zn}$ ) in $\mathrm{Co} /$ metal ratio of $1: 1$. Under these conditions, the coexisting metals did not have any significant impact on the distance of the color band formed. As a validation study of the distance-based $\mu$ PADs, some irrigation water samples were collected (Scottsdale, Tasmania, Australia) and spiked with known concentration (5 ppm) of standard Co solution and then analyzed both with the $\mu \mathrm{PADs}$ and atomic absorption spectroscopy (AAS). The results obtained from both these techniques (Table S-1) were in a very good agreement (within $10 \%$ error). On the other hand, the nonspiked water samples (blank) did not produce any distance signal on the $\mu \mathrm{PADs}$ which showed these samples were at levels below LOD of the target metal. These results were confirmed by the AAS analysis, where the concentrations of the metals in blanks were measured to be at trace levels $(\approx 5 \mathrm{ppb})$ which were below the LOD of the $\mu$ PADs.

\section{CONCLUSIONS}

Several fabrication steps of $\mu \mathrm{PADs}$ can be integrated in a simple manner by coupling a wax printer and an electronic craft plotter/cutter. Wax printing as the most popular technique for creating hydrophobic barriers for $\mu$ PADs is performed by a wax printer and then followed by the cutting and deposition steps done by the plotter/cutter. Optical scanning function of the plotter/cutter is used to ensure the geometrical alignment of the multiple fabrication steps. The accuracy and precision of alignment of wax printing relative to cutting and plotting could be quantified in purposely designed fabrication experiments measuring coordinates of cut and plotted cross-shaped features. Cutting and deposition can be fully automated and be done exactly inside the user-selected spots minimizing misalignment issues and allowing a computerized fabrication process. This approach is illustrated by fabrication of distance-based $\mu$ PADs which need high precision for cutting and deposition steps. The RSD (5.61\%) obtained for determination of cobalt in water samples, indicates the high reliability of this method for fabrication of $\mu$ PADs. The newly developed method can be readily implemented for rapid, low-cost, simple, robust, and reproducible prototyping of different types of $\mu \mathrm{PADs}$ which include multiple steps (e.g., wax printing, cutting, and deposition). However, the fabrication process does not necessarily have to include the combination of steps in a particular order such as here (Figure 1). In other words, any order of these steps can be performed in an equally aligned manner for various applications. For instance, integration of only two steps from the three shown in this work can be used either separately or along with other possible techniques in fabrication of $\mu \mathrm{PADs}$ or other types of miniaturized analytical devices.

\section{ASSOCIATED CONTENT}

\section{S Supporting Information}

The Supporting Information is available free of charge on the ACS Publications website at DOI: 10.1021/acs.analchem.7b03796.

Chemicals and materials, further experimental details for alignment of the fabrication steps, coordinate determination of the center points of plotted crosses, cobalt detection, microscopic measurements, compound graph- ical design for manipulation of plotter/cutter (Figure S1 ), workspace of the Silhouette Studio software (Figure S-2), paper media undergoing four different stages of the fabrication process (Figure S-3), dimensions of the desired cutting, and deposition areas (Figure S-4), front view of the Silhouette Cameo plotter/cutter (Figure S5), registration marks and optical scanner (Figure S-6), sample zone and sample inlet configuration after cutting (Figure S-7), effect of paper available in the sample zone in determination of cobalt (Figure S-8), "Auto Distance Measurement" by DinoCapture 2.0 software (Figure S9), and recovery test of spiked water sample (Table S-1; PDF).

Demonstration of the cutting step in fabrication process of $\mu$ PADs (Movie S-1; MPG).

Demonstration of the deposition step in fabrication process of $\mu \mathrm{PADs}$ (Movie S-2; MPG).

\section{AUTHOR INFORMATION}

\section{Corresponding Author}

*Fax: +61 362262858. E-mail: mirek.macka@utas.edu.au.

ORCID

Brett Paull: 0000-0001-6373-6582

Mirek Macka: 0000-0002-6792-2574

\section{Notes}

The authors declare no competing financial interest.

\section{ACKNOWLEDGMENTS}

M.M. gratefully acknowledges the Australian Research Council Future Fellowship (FT120100559).

\section{REFERENCES}

(1) Cate, D. M.; Adkins, J. A.; Mettakoonpitak, J.; Henry, C. S. Anal. Chem. 2015, 87, 19-41.

(2) Meredith, N. A.; Quinn, C.; Cate, D. M.; Reilly, T. H.; Volckens, J.; Henry, C. S. Analyst 2016, 141, 1874-1887.

(3) Yang, Y.; Noviana, E.; Nguyen, M. P.; Geiss, B. J.; Dandy, D. S.; Henry, C. S. Anal. Chem. 2017, 89, 71-91.

(4) He, Y.; Wu, Y.; Fu, J. Z.; Wu, W. B. RSC Adv. 2015, 5, 7810978127.

(5) Xia, Y.; Si, J.; Li, Z. Biosens. Bioelectron. 2016, 77, 774-789.

(6) Tenda, K.; Ota, R.; Yamada, K.; Henares, T. G.; Suzuki, K.; Citterio, D. Micromachines 2016, 7, 80.

(7) Nuchtavorn, N.; Macka, M. Anal. Chim. Acta 2016, 919, 70-77.

(8) Yamada, K.; Henares, T. G.; Suzuki, K.; Citterio, D. Angew. Chem., Int. Ed. 2015, 54, 5294-5310.

(9) de Oliveira, R. A.; Camargo, F.; Pesquero, N. C.; Faria, R. C. Anal. Chim. Acta 2017, 957, 40-46.

(10) Glavan, A. C.; Martinez, R. V.; Maxwell, E. J.; Subramaniam, A. B.; Nunes, R. M.; Soh, S.; Whitesides, G. M. Lab Chip 2013, 13, 2922-2930.

(11) da Silva, E. T.; Santhiago, M.; de Souza, F. R.; Coltro, W. K.; Kubota, L. T. Lab Chip 2015, 15, 1651-1655.

(12) Koo, Y.; Shanov, V. N.; Yun, Y. Micromachines 2016, 7, 72.

(13) Phan, D. T.; Shaegh, S. A. M.; Yang, C.; Nguyen, N. T. Sens. Actuators, B 2016, 222, 735-740.

(14) Afonso, A. S.; Uliana, C. V.; Martucci, D. H.; Faria, R. C. Talanta 2016, 146, 381-387.

(15) Oyola-Reynoso, S.; Heim, A. P.; Halbertsma-Black, J.; Zhao, C.; Tevis, I. D.; Çınar, S.; Cademartiri, R.; Liu, X.; Bloch, J. F.; Thuo, M. M. Talanta 2015, 144, 289-293.

(16) Li, W.; Shi, Z.; Fang, C.; Lu, Y.; Yu, L.; Li, C. M. Microfluid. Nanofluid. 2017, 21, 71.

(17) Amin, R.; Ghaderinezhad, F.; Li, L.; Lepowsky, E.; Yenilmez, B.; Knowlton, S.; Tasoglu, S. Anal. Chem. 2017, 89, 6351. 
(18) Tian, T.; Li, J.; Song, Y.; Zhou, L.; Zhu, Z.; Yang, C. J. Lab Chip 2016, 16, 1139-1151.

(19) Cate, D. M.; Dungchai, W.; Cunningham, J. C.; Volckens, J.; Henry, C. S. Lab Chip 2013, 13, 2397-2404.

(20) Cate, D. M.; Noblitt, S. D.; Volckens, J.; Henry, C. S. Lab Chip 2015, 15, 2808-2818.

(21) Pratiwi, R.; Nguyen, M. P.; Ibrahim, S.; Yoshioka, N.; Henry, C. S.; Tjahjono, D. H. Talanta 2017, 174, 493-499.

(22) Yamada, K.; Henares, T. G.; Suzuki, K.; Citterio, D. ACS Appl. Mater. Interfaces 2015, 7, 24864-24875.

(23) Wei, X.; Tian, T.; Jia, S.; Zhu, Z.; Ma, Y.; Sun, J.; Lin, Z.; Yang, C. J. Anal. Chem. 2016, 88, 2345-2352.

(24) Zhang, Y.; Gao, D.; Fan, J.; Nie, J.; Le, S.; Zhu, W.; Yang, J.; Li, J. Biosens. Bioelectron. 2016, 78, 538-546.

(25) Sarkar, B. Heavy Metals in the Environment; CRC Press, 2002.

(26) Underwood, E. Trace Elements in Human and Animal Nutrition, 4th ed.; Elsevier, 2012.

(27) ANZECC, ARMCANZ (Australian and New Zealand Environmental and Conservation Council, Agriculture and Resource Management Council of Australia and New Zealand, Canberra) Australian and New Zealand Guidelines for Fresh and Marine Water Quality, No. 4; ANZECC, 2000; 3, Chapter 9.

(28) Safavi, A.; Abdollahi, H.; Nezhad, M. H.; Kamali, R. Spectrochim. Acta, Part A 2004, 60, 2897-2901.

(29) Barbosa, L. C.; Maltha, C. R.; Demuner, A. J.; Cazal, C. M.; Reis, E. L.; Colodette, J. L. BioResources 2012, 8, 1043-1054.

(30) Murphy, A.; Gorey, B.; De Guzman, K.; Kelly, N.; Nesterenko, E. P.; Morrin, A. RSC Adv. 2015, 5, 93162-93169. 